\title{
Dynamics of Plasma Lipoproteins and Lipids During Double Filtration Plasmapheresis (DEP)
}

\author{
Shigeki Sakata, Takashi Komaki, Noriko Kojima, Masafumi Matsuda, \\ Satoshi Maeda, Masaka Kawade* and Kiyoshi Miura
}

\begin{abstract}
Plasma lipids and lipoproteins obtained from different places of the circuit of double filtration plasmapheresis (DFP) were measured and effect of DFP therapy on removal of them was examined. When 2A was used as a second filter, $69.8 \%, 52.4 \%, 63.0 \%, 58.0 \%, 60.8 \%, 59.2 \%$ and $63.9 \%$, respectively of $\beta$ lipoprotein, cholesterol, triglyceride, high density lipoproteins (HDL), phospholipids, free cholesterol and lipoprotein (a) (Lp(a)) were removed from the patient's plasma. When $4 \mathrm{~A}$ was used as a second filter, $69.0 \%, 56.8 \%, 53.2 \%, 45.4 \%, 56.0 \%, 50.9 \%$ and $51.7 \%$, respectively of $\beta$-lipoprotein, cholesterol, triglyceride, HDL, phospholipids, free cholesterol and $\mathrm{Lp}(\mathrm{a})$ were removed from the patient's plasma. In contrast, concentrations of free fatty acids (FFA) after DFP therapy using filter 4A and $2 \mathrm{~A}$ increased to $222.8 \%$ and $256.4 \%$, respectively. Thus, it was shown that except for FFA, DFP therapy using either $2 \mathrm{~A}$ or $4 \mathrm{~A}$ as a second filter is effective in reducing concentrations of plasma lipids and lipoproteins.
\end{abstract}

Key Words: Double filtration plasmapheresis, Plasma lipids and lipoproteins.

Therapeutic plasmapheresis has been introduced for the treatment of various kinds of recalcitrant diseases including familial hypercholesterolemia. In 1975, Thompson et al. ${ }^{1)}$ first treated patients with familial hypercholesterolemia with plasma exchange, by substituting plasma removed from patients with human plasma albumin. Since then, various trials involving removal of lipids from the sera of patients with hyperlipemia have been reported ${ }^{2-4)}$.

Concerning the economy of plasma lipids and lipoproteins during DFP therapy, little has been so far reported. We have treated 11 patients with rheumatoid arthritis (RA), systemic lupus erythematosus (SLE), mixed connective tissue disease (MCTD), progressive systemic sclerosis (PSS), Graves' disease and cold urticaria with DFP, and the effect of these treatments on the removal of lipoproteins and lipids from each patient was examined.

\section{MATERIALS AND METHODS}

Heparinized blood was obtained from the femoral vein of each patient (Table 1) and passed through the first filter (Plasmacure, Kuraray Co., Osaka) of DFP monitor (KM-8500, Kuraray Co., Osaka) where cellular and plasma components were separated. Plasma components thus obtained (Fig. 1, A) were then passed through the second filter (Evaflux 4A or 2A, Kuraray Co., Osaka) in which macromolecules such as IgM and IgG immune complexes were selectively removed and discarded (Fig. 1, C). Each patient's plasma thus removed of its harmful components (Fig. 1, B) was returned together with cellular components to the same patient through either brachial or femoral vein. In order to avoid hypoproteinemia and hypovolemia due to the discard of unnecessary plasma components during filtration, a $5 \%$ albumin or plasma protein fraction (PPF) of equal volume to

From The Third Department of Internal Medicine and *Department of Laboratory Medicine,

Gifu University School of Medicine, Gifu 500, Japan.

Received for publication August 25, 1986.

Reprint requests to: Shigeki Sakata, MD, The Third Department of Internal Medicine,

Gifu University School of Medicine, 40, Tsukasa-cho, Gifu 500, Japan. 
Table 1. List of the patients who underwent DFP therapy. SLE; systemic lupus erythematosus, MCTD; mixed connective tissue disease, RA; rheumatoid arthritis, PSS; progressive systemic sclerosis

$\begin{array}{rccl}\text { Case } & \text { Sex } & \begin{array}{c}\text { Age } \\ \text { (years) }\end{array} & \text { Diagnosis } \\ 1 . & & 52 & \text { SLE } \\ 2 . & \mathrm{F} & 52 & \text { SLE } \\ 3 . & \mathrm{F} & 17 & \text { MCTD } \\ 4 . & \mathrm{M} & 35 & \text { Cold urticaria } \\ 5 . & \mathrm{F} & 52 & \text { RA } \\ 6 . & \mathrm{F} & 62 & \text { RA } \\ 7 . & \mathrm{F} & 21 & \text { RA } \\ 8 . & \mathrm{F} & 54 & \text { RA } \\ 9 . & \mathrm{F} & 43 & \text { RA } \\ 10 . & \mathrm{F} & 55 & \text { PSS, SLE } \\ 11 . & \mathrm{F} & 60 & \text { Graves' disease }\end{array}$

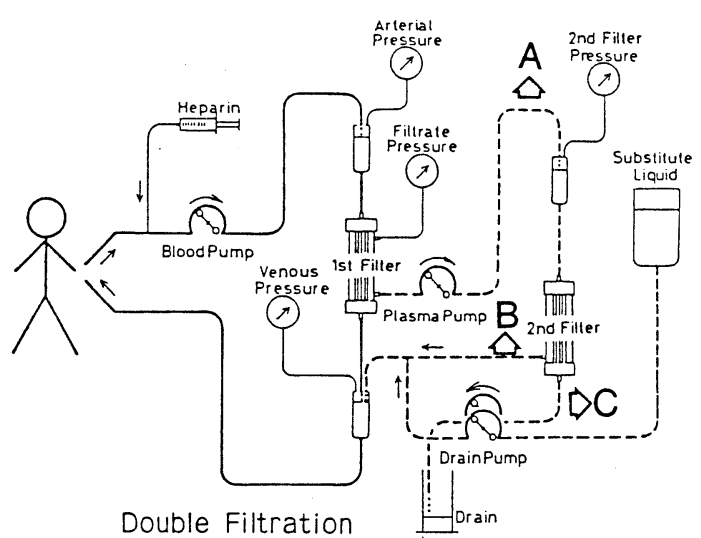

Fig. 1. Schematic representation of DFP. Arrows (A, B and $\mathrm{C}$ ) indicate the points where an aliquot of plasma was taken for the present investigation.

the discarded plasma was infused intravenously during DFP therapy (Fig. 1).

During DFP therapy, each aliquot of plasma was obtained from the three points of the circuit (Fig. 1, A, B and C) and concentrations of $\beta$-lipoprotein, phospholipids, free cholesterol, and FFA were measured using automatic analyzer (enzymatic method, Greiner selective analyzer, Electronics 11D, Lagenthal, Switzerland). Concentrations of cholesterol and triglyceride were measured by enzymatic method using automatic analyzer (Hitachi 726). HDL was also measured by enzymatic method after precipitation of $\beta$-lipoprotein
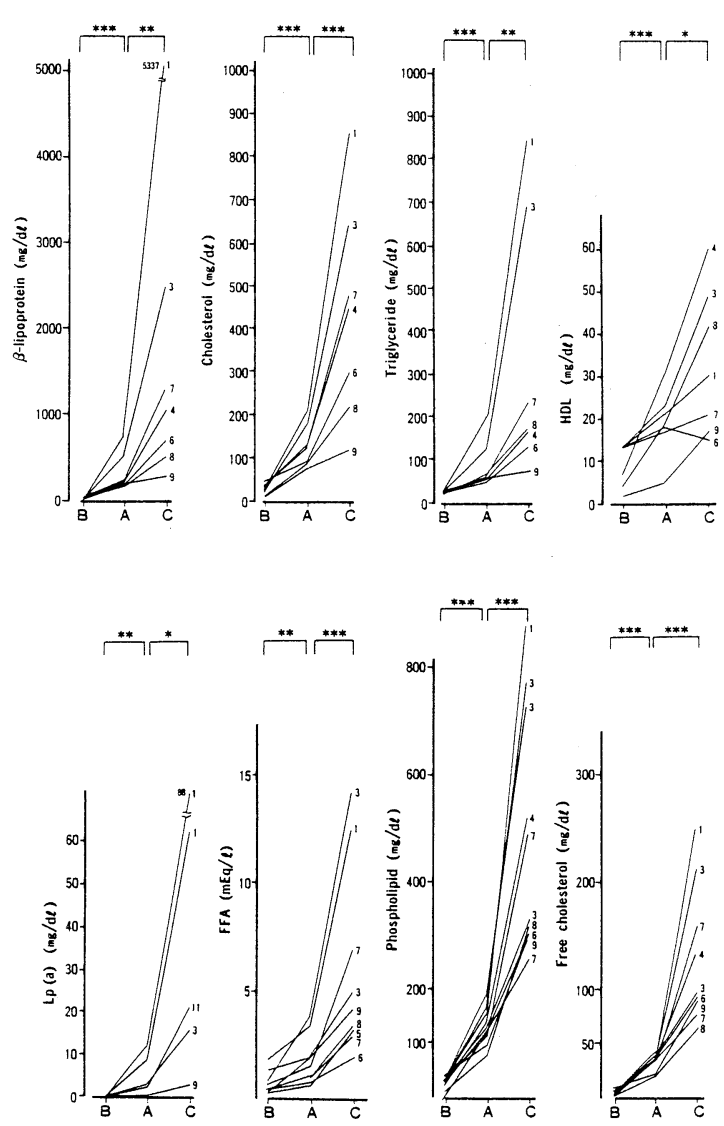

Fig. 2. Concentrations of plasma $\beta$-lipoprotein, cholesterol, triglyceride, HDL, Lp(a), FFA, phospholipids and free cholesterol obtained from three different points (A, B and C) of circuit during DFP therapy using $2 \mathrm{~A}$ as a second filter. Number in this figure indicates patient's number as listed in Table 1 .

$* \mathrm{P}<0.05, * * \mathrm{P}<0.01, * * * \mathrm{P}<0.001$, n.s., not significant.

using $\mathrm{Mg}^{2+} /$ dextran sulfate. $\beta$-lipoprotein was measured by turbidimetric method and $\mathrm{Lp}(\mathrm{a})$ was measured according to the reported method ${ }^{5)}$. The effect of DFP therapy on the removal of plasma lipoproteins and lipids from the plasma was examined. Statistical analyses were done by the paired t-test.

\section{RESULTS}

1. Concentrations of $\beta$-lipoprotein, cholesterol, triglyceride, HDL, Lp(a), FFA, phospholipids, and free cholesterol obtained from three different points of the circuit during DFP treatment (Figs. 2, 3) 

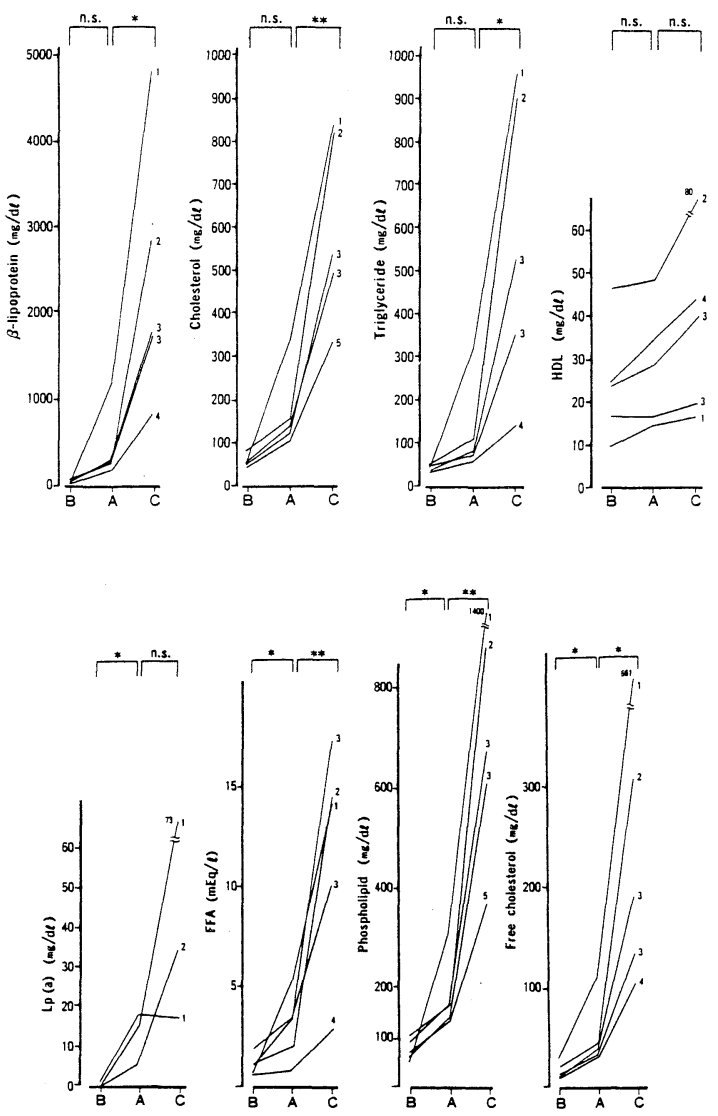

Fig. 3. Concentrations of plasma $\beta$-lipoprotein, cholesterol, triglyceride, HDL, Lp(a), FFA, phospholipids and free cholesterol obtained from three different points (A, B and C) of circuit during DFP therapy using $4 \mathrm{~A}$ as a second filter. Number in this figure indicates patient's number as listed in Table 1 .

$* \mathrm{P}<0.05, * * \mathrm{P}<0.01$, n.s., not significant.

As shown in Fig. 2, when $2 \mathrm{~A}$ was used as a second filter, concentrations of plasma $\beta$-lipoprotein, cholesterol, triglyceride, HDL, Lp(a), FFA, phospholipids, and free cholesterol obtained from point A were significantly lower than those obtained from point $\mathrm{C}$, respectively, and those obtained from point $B$ were significantly lower than those obtained from point $\mathrm{A}$. When $4 \mathrm{~A}$ was used as a second filter, concentrations of plasma $\beta$-lipoprotein, cholesterol, triglyceride, FFA, phospholipids, and free cholesterol obtained from point A were significantly lower than those obtained from point C, respectively. Concentrations of HDL and Lp(a) were not significantly different in these two points. Plasma Lp(a), FFA, phospholipids, and free cholesterol obtained from point A were significantly higher than those obtained from point $B$, whereas such significant difference was not observed in concentrations of $\beta$-lipoprotein, cholesterol, and HDL.

\section{Clearance rate of lipoproteins and lipids after} DFP therapy (Table 2)

Concentrations of lipoproteins and lipids were compared before and immediately after DFP therapy and clearance rate of each lipoprotein and lipid was calculated and summarized in Table 2. When $2 \mathrm{~A}$ was used as a second filter, $69.8 \%, 52.4 \%$, $63.0 \%, 58.0 \%, 63.9 \%, 60.9 \%$, and $59.2 \%$ of $\beta$-lipoprotein, cholesterol, triglyceride, HDL, Lp(a), phospholipids, and free cholesterol, respectively, were removed from the patient's plasma. When $4 \mathrm{~A}$ was used as a second filter, $69.0 \%, 56.8 \%, 53.2 \%$, $45.4 \%, 51.7 \%, 56.0 \%$, and $50.9 \%$ of $\beta$-lipoprotein, cholesterol, triglyceride, HDL, Lp(a), phospholipids, and free cholesterol, respectively, were removed from the patient's plasma. Plasma concentration of FFA after DFP therapy using 2A or $4 \mathrm{~A}$ increased to $222.8 \%$ or $256.4 \%$, respectively.

Table 2. Clearance rate of lipoporteins and lipids after DFP therapy

$\begin{array}{lccc}\begin{array}{c}\text { Lopoproteins } \\ \text { or lipids }\end{array} & \begin{array}{c}\text { Second } \\ \text { filter used }\end{array} & \begin{array}{c}\text { Number } \\ \text { of cases }\end{array} & \%^{*} \\ \beta \text {-lipoprotein } & 2 \mathrm{~A} & 7 & 69.8 \pm 13.3 \\ & 4 \mathrm{~A} & 2 & 69.0 \pm 13.0 \\ \text { cholesterol } & 2 \mathrm{~A} & 7 & 52.4 \pm 15.0 \\ & 4 \mathrm{~A} & 2 & 56.8 \pm 5.6 \\ \text { triglyceride } & 2 \mathrm{~A} & 7 & 63.0 \pm 20.3 \\ & 4 \mathrm{~A} & 2 & 53.2 \pm 13.8 \\ \text { HDL } & 2 \mathrm{~A} & 6 & 58.0 \pm 10.2 \\ & 4 \mathrm{~A} & 2 & 45.4 \pm 6.3 \\ \text { Lp (a) } & 2 \mathrm{~A} & 4 & 63.9 \pm 28.7 \\ & 4 \mathrm{~A} & 3 & 51.7 \pm 16.5 \\ \text { FFA } & 2 \mathrm{~A} & 5 & 222.8 \pm 176.1 \\ & 4 \mathrm{~A} & 3 & 256.4 \pm 119.9 \\ \text { phospholipids } & 2 \mathrm{~A} & 5 & 60.9 \pm 26.2 \\ & 4 \mathrm{~A} & 3 & 56.0 \pm 22.2 \\ \text { free cholesterol } & 2 \mathrm{~A} & 5 & 59.2 \pm 25.2 \\ & 4 \mathrm{~A} & 3 & 50.9 \pm 31.4\end{array}$

*Values (\%) were calculated by dividing concentrations of plasma lipids and lipoproteins before DFP therapy by those after DFP therapy. 


\section{DISCUSSION}

In our present investigation, concentrations of plasma $\beta$-lipoprotein, cholesterol, triglyceride, HDL, Lp(a), FFA, phospholipids, and free cholesterol were measured in the plasma obtained from three different points of the circuit during DFP therapy and the efficacy of DFP in the removal of plasma lipoproteins and lipids was examined. When 2A was used as a second filter, concentrations of $\beta$-lipoprotein, cholesterol, triglyceride, HDL, Lp(a), FFA, phospholipids, and free cholesterol obtained from point $\mathrm{C}$ were significantly higher than those obtained from point $\mathrm{A}$, and those obtained from A were significantly higher than those obtained from $\mathrm{B}$. When $4 \mathrm{~A}$ was used as a second filter, concentrations of $\beta$-lipoprotein, cholesterol, triglyceride, FFA, phospholipids, and free cholesterol obtained from point $\mathrm{C}$ were significantly higher than those obtained from point A, whereas no significant difference was observed in concentrations of HDL and $L p(a)$ obtained from these two points. Concentrations of $\operatorname{Lp}(\mathrm{a})$, FFA, phospholipids, and free cholesterol obtained from point $\mathrm{A}$ were higher than those obtained from point $\mathrm{B}$. Concerning the plasma concentrations of $\beta$-lipoprotein, cholesterol, triglycerides, and HDL, although concentrations in point A were higher than point $\mathrm{B}$ in every case, the difference was statistically insignificant. These results indicate the effective removal of lipids and lipoproteins by the second filter. Thus, after single DFP therapy using $2 \mathrm{~A}$ or $4 \mathrm{~A}$ as a second filter, approximately $30-50 \%$ of plasma lipids and lipoproteins are removed from plasma. The reason why plasma FFA increased more than twice prior to DFP therapy, despite effective removal from the circuit, is probably due to the effect of constant infusion of heparin (2000-3000 U/hour) to avoid blood coagulation during DFP therapy.

\section{REFERENCES}

1) Thompson GR, Lowenthal R and Myant NB: Plasma exchange in the management of homozygous familiar hypercholesterolemia. Lancet ii: 1208, 1975.

2) Yokoyama S: Plasmapheresis treatment of familiar hypercholesterolemia. Pharma Medica 2: 89, 1984.

3) Lupien PJ, Moorjani S, Lou M, Brun D and Gagne CI: Removal of cholesterol from blood by affinity binding to heparin agarose: Evaluation on treatment in homozygous familiar hypercholesterolemia. Pediatr Res 14: 113, 1980.

4) Graisely B, Cloarec M and Salmon S: Extracorporeal plasma therapy for homozygous familiar hypercholesterolemia. Lancet ii: 1147, 1980.

5) Albers JJ and Hazzard WR: Immunological quantification of human plasma $\mathrm{Lp}(\mathrm{a})$ lipoprotein. Lipids 9: 15,1974 . 